\title{
Unstable Graves' disease as a precipitating factor for cerebral sinus venous thrombosis
}

\author{
Karolina Anuszkiewicz ${ }^{(1)}$, Anna Szerszenowicz ${ }^{\circledR 1}$, Kamil Dzwilewski ${ }^{1}$, Nicole Geryk ${ }^{1}$, \\ Marta Zawadzka ${ }^{\circledR 1}$, Julia Radoń-Proskura ${ }^{\circledR 2}$, Maria Mazurkiewicz-Bełdzińska ${ }^{\circledR 1}$ \\ ${ }^{1}$ Department of Developmental Neurology, Medical University of Gdansk, Gdansk, Poland \\ ${ }^{2}$ Department of Paediatrics, Haematology, and Oncology, Medical University of Gdansk, Gdansk, Poland
}

Key words: cerebral sinus venous thrombosis; CSVT; Graves' disease; hyperthyroidism; child

Cerebral sinus venous thrombosis (CSVT) is a type of ischaemic stroke, mainly seen in middle-aged females; however, it is also observed in the paediatric population. Risk factors vary from those in the adult population - the most common in children are infections in the head and neck area, dehydration, head trauma, hypercoagulable states and chronic states such as nephrotic syndrome, lupus or liver disease [1]. Several articles describe the connection between hyperthyroidism and thrombotic complication [2]. Precise knowledge of CSVT risk factors is important because the clinical presentation is often unspecific.

A 15-year-old male patient experienced headache and progressive paresis of the right upper and lower limb for two days. Medical history revealed a diagnosis of Graves' disease, which was made three years before, treated with oral thiamazole $5 \mathrm{mg}$ twice daily. On admission the patient was conscious, but without logical contact, tachycardia with heart rate 130-150 bpm was present, and blood pressure was within the normal range for age. Neurological examination revealed motor aphasia and right-sided hemiparesis. During the first days of hospitalization focal to bilateral tonic-clonic seizures occurred.

Brain magnetic resonance (MR) revealed thrombosis of the superior sagittal sinus as well as left transverse and sigmoid sinuses with the bulb of left jugular vein (Fig. 1). Parallel haemorrhage due to venous infarction in the left precentral region of the brain $(10 \times 4 \mathrm{~mm})$ was observed.

Laboratory results including full blood count, C-reactive protein (CRP), creatinine, and liver enzymes were normal. Coagulation screening tests of activated partial thromboplastin time, prothrombin time, thrombin time, level of antithrombin, factor $\mathrm{XI}$, protein $\mathrm{C}$

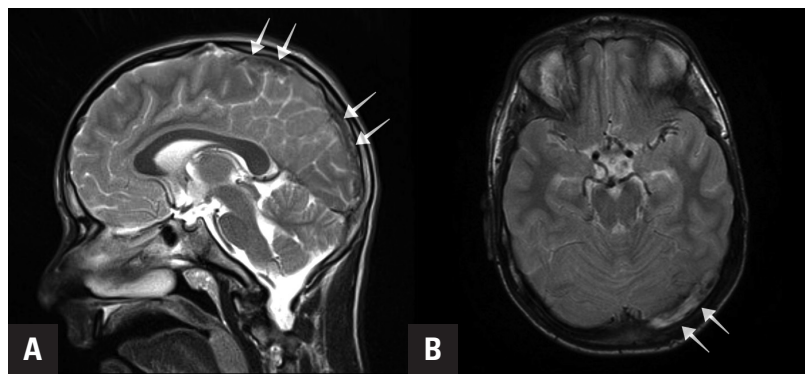

Figure 1. MR on admission date: A. T2 sequence with upper sagittal sinus thrombosis; B. T1 sequence with left transverse sinus thrombosis (marked by arrows)

and S, as well as free protein S, homocysteine, and lipoprotein(a) were within laboratory ranges. Analyses of genetic polymorphisms of factor V Leiden G1691A and factor II G20210A were negative.

The levels of fVIII and vWf as well as the activity of vWf were significantly increased - 405\% [normal range (NR): $70-150 \%$ ], 205\% (NR: 50-160\%), and $274 \%$ (NR: 60-170\%) respectively. There was a moderate rise in factor IX level (137\%; NR:70-120\%) and fibrinogen $(4.33 \mathrm{~g} / \mathrm{L}$; NR: $1.80-3.50 \mathrm{~g} / \mathrm{L})$. Laboratory methods utilized one-stage clotting assay with factor deficient plasma, ristocetin cofactor assay (vWF:RCo) for activity of $\mathrm{vWf}$, and immunoturbidometric assay for von vWf level. Systemic lupus erythematosus (SLE) and antiphospholipid syndrome (APS) were excluded based on Systemic Lupus International Collaborating Clinics (SLICC 2012) and APS criteria, respectively. Anti-neutrophil cytoplasmic antibodies (ANCAs) were absent.

Thyroid workup was consistent with Graves' disease. This included a low level of thyroid-stimulating hormone (TSH): $0.007 \mathrm{uU} / \mathrm{mL}$, high free triiodothyro- 
nine (fT3): $9.06 \mathrm{pmol} / \mathrm{l}$, and high free thyroxine (fT4): $33 \mathrm{pmol} / \mathrm{L}$. Level of antibodies against TSH receptor (TRAb) was $30.66 \mathrm{IU} / \mathrm{L}$, antibodies against thyroglobulin (TG) was $286 \mathrm{IU} / \mathrm{L}$, and antibodies against thyroid peroxidase (TPO) was $742 \mathrm{IU} / \mathrm{L}$. Thyroid ultrasound confirmed enlargement of the gland; the thyroid gland volume was $43.2 \mathrm{~mL}$. Transthoracic echocardiogram did not reveal any features of heart defect.

The patient was anticoagulated with unfractionated heparin intravenously and transitioned to enoxaparin under the control of anti-Xa. Due to Graves' disease exacerbation, the dose of thiamazole was increased to $10 \mathrm{mg}$ three times per day. Propranolol was applied to reduce the hyperthyroidism symptoms. Concomitant seizures were treated with clonazepam for a week. Later the seizures were not observed.

Over the next two weeks, successive regression of neurological symptoms was observed. Follow-up brain MRIs after 2 weeks and 3 months revealed significant reduction of thrombus and haemorrhage size. The levels of fT3 and fT4 decreased to $6.29 \mathrm{pmol} / \mathrm{L}$ and 10.63 $\mathrm{pmol} / \mathrm{L}$, respectively. Good outcome of hyperthyroidism treatment led to decreased vWF (81\%) and fVIII $(161 \%)$. The patient was discharged in a good general condition, walking by himself, with little evidence of paraplegia, and he was recommended further interdisciplinary treatment (enoxaparin, thiamazole, and rehabilitation).

The data describing precisely the effect of elevated thyroid hormones leading to thrombosis are limited. In the course of hyperthyroidism the level of catecholamines is raised and leads to increased activity of factor VIII, IX, fibrinogen, and vWf with the result of accelerated platelet plug formation [3, 4]. Another path implies decreased fibrinolytic activity compared with endothelial activation. Graves' disease results from the presence of anti-thyroid stimulating hormone receptor antibodies. Rehman et al. suggest that it may be con- nected with an increased risk of CSVT due to abnormal activation of thrombocytes by the antibodies and the dysfunction of vascular endothelium [5]. However, no paper to this day has unambiguously recognized this disease as a possible cause of thrombosis.

The patient was eventually diagnosed with unstable hyperthyroidism, which could have led to hypercoagulation. It is important to notice that other factors could play a role such as venous stasis due to thyroid gland enlargement or dehydration related to hyperthyroidism. No other risk factors of thrombosis or stroke were recognized in our patient, and the family history was negative.

Cerebral sinus venous thrombosis and stroke in the course of hyperthyroidism in paediatric patients is a rare complication within this age group. Therefore, particular attention should be paid to CSVT in the event of unstable hyperthyroidism and non-specific neurological symptoms among children with Graves' disease.

\section{Funding}

Nothing to declare.

\section{Conflict of interests}

None declared.

\section{References}

1. Capecchi M, Abbattista M, Martinelli I. Cerebral venous sinus thrombosis. J Thromb Haemost. 2018; 16(10): 1918-1931, doi: 10.1111/jth.14210, indexed in Pubmed: 29923367.

2. Hieber M, von Kageneck C, Weiller C, et al. Thyroid Diseases Are an Underestimated Risk Factor for Cerebral Venous Sinus Thrombosis. Front Neurol. 2020; 11: 561656, doi: 10.3389/fneur.2020.561656, indexed in Pubmed: 33192995.

3. Waheed W, Aljerdi S, Decker B, et al. Cerebral venous thrombosis associated with thyrotoxicosis, the use of desmopressin and elevated factor VIII/von Willebrand factor. BMJ Case Rep. 2016; 2016, doi: 10.1136/bcr-2016-216584, indexed in Pubmed: 27503942.

4. Dai A, Wasay M, Dubey N, et al. Superior sagittal sinus thrombosis secondary to hyperthyroidism. J Stroke Cerebrovasc Dis. 2000; 9(2): 89-90, doi: 10.1053/jscd.2000.0090089, indexed in Pubmed: 17895204.

5. Rehman A, Husnain MG, Mushtaq K, et al. Cerebral venous sinus thrombosis precipitated by Graves disease. BMJ Case Rep. 2018; 2018, doi: 10.1136/bcr-2017-224143, indexed in Pubmed: 29866676. 